Philippe Cordez

\title{
Materielle Metonymie. \\ Thomas von Cantimpré und das erste Horn des Einhorns
}

„Präparate bilden nichts ab, sie sind, wenn man so will, ,Bilder“ ihrer selbst: materielle Metonymien.“ So führte Hans-Jörg Rheinberger in der zweiten Ausgabe der Bildwelten des Wissens eine Dinggattung ein, die in den Naturwissenschaften eine entscheidende Rolle spielt. ${ }^{1}$ Der lateinischen Etymologie nach ist ein Präparat (praeparatum) etwas Zu- bzw. Vor-Bereitetes oder auch Vor-Gestelltes: Etwas, dessen Status dadurch bestimmt ist, dass es aus einem anderen materiellen Zusammenhang als seinem aktuellen stammt, in dem es sich selbst repräsentiert. Dieses Prinzip ist das einer Metonymie: Ein Ereignis der Sprache, in dem ein Objekt mit einem Wort genannt wird, das eigentlich ein anderes Objekt bezeichnet, welches aber mit dem ersten durch eine konstante sachliche Beziehung verbunden ist. Das Wort rutscht sozusagen entlang dieser Verbindung. So kann zum Beispiel ein Tier, dessen Anatomie sich dadurch auszeichnet, dass sein Kopf ein Horn trägt, mit dem Ausdruck „ein Horn“ bezeichnet werden - das Einhorn. Das Horn ist die Metonymie des Einhorns, und wer dem Horn eines Einhorns begegnet, was viele im Mittelalter und in der Frühen Neuzeit erlebt haben, hat eine materielle Metonymie vor sich - also ein Präparat.

Dieser Befund stellt freilich die Frage nach Geschichte und Funktionen des Präparats. Der Duden (2007) nennt „Forschung u. Lehre“ als seine Zwecke. Petra Lange-Berndt hat gezeigt, wie präparierte Tiere ihren Einzug in der Kunstwelt gehalten und dort, besonders in den 1990er-Jahren, einer Kritik an den Behauptungen der Naturwissenschaft zur Authentizität und Natürlichkeit physischer Körper gedient haben. ${ }^{2}$ Wird nun das Forschungsfeld auf die breite Vielfalt der menschlichen Gesellschaften und Techniken erweitert, können Präparate in reicher Vielfalt erkannt werden: die Beuteattrappen eines Jägers, das Federarrangement am Hut einer Eleganten, das Bärenkostüm eines Schamanen, ein Jaguarzahn als Amulett, der Schädel eines Heiligen in seinem Reliquiar usw. All diese Objekte stellen das, was sie waren und in präparierter Form noch sind, in einem neuen Zusammenhang dar und erfüllen hiermit unterschiedliche Funktionen. Präparate ergeben sich aus der materiellen und intellektuellen, bzw. technischen und zugleich kulturellen Verarbeitung der Natur durch den Mensch. Sie sind in diesem Sinne Produkte und Zeugen einer humanen Sozialisierung der Tier- bzw. Menschenleichen, oder noch der Pflanzen, der Steine, ihrer einzelnen Moleküle usw. Im Folgenden soll

1 Hans-Jörg Rheinberger: Präparate -,Bilder' ihrer selbst. Eine bildtheoretische Skizze. In: Bildwelten des Wissens, Band 1,2 (Oberflächen der Theorie), Berlin 2003, S. 9-19.

2 Petra Lange-Berndt: Animal Art. Präparierte Tiere in der Kunst 1850-2000, München 2009. 


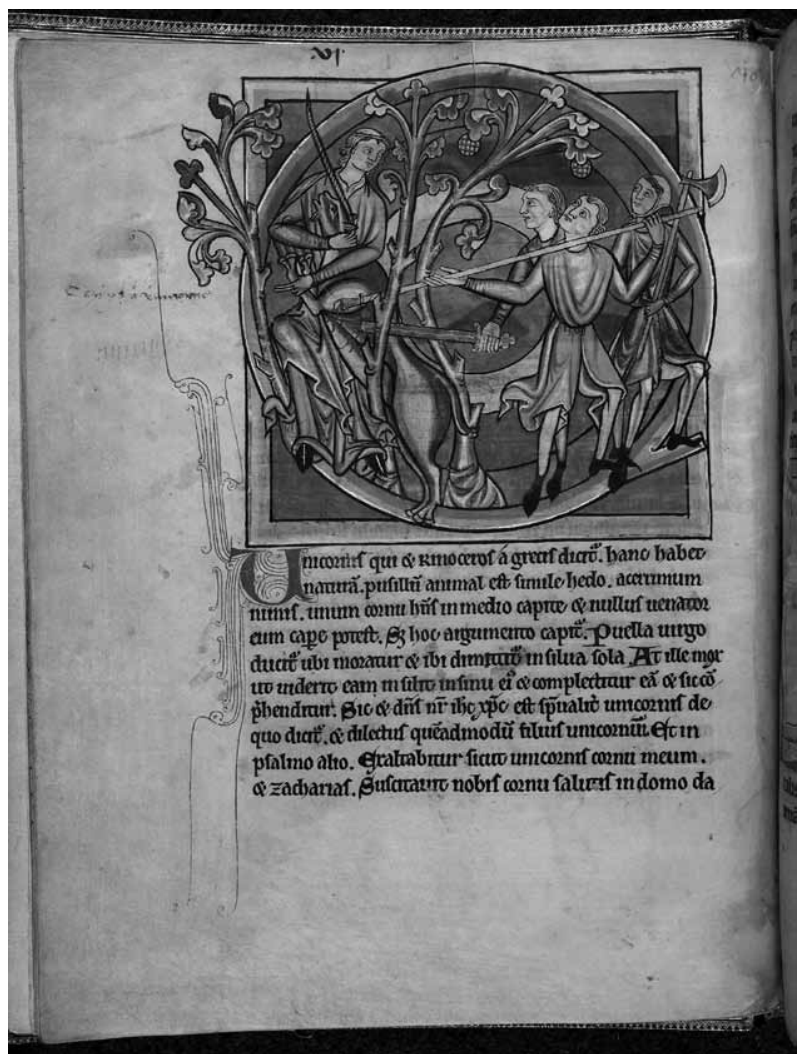

1: Einhornjagd. Bestiarium, Südengland, erste Hälfte des 13. Jahrhunderts. die Genese eines solchen Objekttyps untersucht werden, der mit einem Höhepunkt im 16. Jahrhundert ein besonders hohes Prestige genoss, bis er nach heftiger wissenschaftlicher Debatte für nichtig erklärt wurde. Einhörner existieren natürlich nicht, aber das Einhorn-Horn war trotzdem ein Präparat. Wie wurde es präpariert? War es ein Horn von Nichts, wie ein Anonymus 1583 fragte - „une corne de néant"? 3 Wovon war es das „,Bild' seiner selbst“? Und was erfährt man anhand dieses paradox anmutenden Falls über Präparate im Allgemeinen?

Der Historiker trifft zum ersten Mal auf das Einhorn-Hornl im „Buch der Bienen“, das Thomas von Cantimpré (ca. 1201-ca. 1270/1272) um 1260 verfasste: Es ist „sieben Fuß lang, wie wir es in der Kirche von Brügge in Flandern sehen [...]“.4 Was war dieses Objekt? Seine Länge, umgerechnet etwa zwei bis zweieinhalb Meter, deutet schon auf den großen Zahn des Narwals, der in den folgenden Jahrhunderten oft als Horn des Einhorns vorgestellt wurde. Er ist mit seiner langen, weißen, aufrechten und spiralartigen Form zum Beispiel auch in zwei Miniaturen eines südenglischen Tierbuches aus der ersten Hälfte des 13. Jahrhunderts zu erkennen. ${ }^{5}$ 入 Abb. 1, Tafel 8, 入 Abb. 2 Solche Bilder ersetzen frühere Darstellungen von Einhörnern mit verschiedenen Hornarten, mitunter auch kurzen und krummen. Sie erscheinen zunächst in England, verbreiten sich am Anfang des 14. Jahrhunderts bis auf den Kontinent und sind Indikatoren für die Erfolgsgeschichte der Identifikation des Narwalzahns mit dem Einhorn-

3 Anonymus: Response au Discours d'Ambroise Paré, touchant l'usage de la licorne..., Paris 1583, S. 9.

4 Thomas Cantimpratensis: Bonum universale de apibus, 1. 2, c. 29, 32, Douai 1605, S. 313. Diese Fallstudie beruht auf meiner Dissertation „Trésor, mémoire, merveilles. Les objets des églises au Moyen Âge“, Paris/Berlin 2010, dort S. 168-251 zu Tierobjekten in mittelalterlichen Kirchen.

5 British Library, London, Harley 4751, fol. 6v. u. 15r. 
Horn. ${ }^{6}$ Der Narwal an sich ist ein vier bis fünf Meter großer Wal aus der Arktis. Den männlichen Walen ragt dieser riesige Zahn üblicherweise links durch die obere Lippe. Gelegentlich wachsen einem Narwal zwei solcher Zähne. Es kommt ebenfalls vor, dass auch ein weiblicher Narwal damit versehen ist. Die Zähne der nahe der Eisbank harpunierten Narwale haben die Inuits bis ins 20. Jahrhundert, wie auch vieles sonst von diesem Tier, für vielfältige Zwecke benutzt. ${ }^{7}$

Doch zurück zum mittelalterlichen Exemplar in Brügge. Die Stadt hatte einen florierenden Hafen an der Nordsee. Daher ist es gut denkbar, dass Seeleute den Zahn eines Narwals in eine der dortigen Kirchen gebracht hatten. Man musste nicht in Grönland gewesen sein: Händler konnten vermittelt haben, außerdem kam damals das Tier wohl öfter und weiter

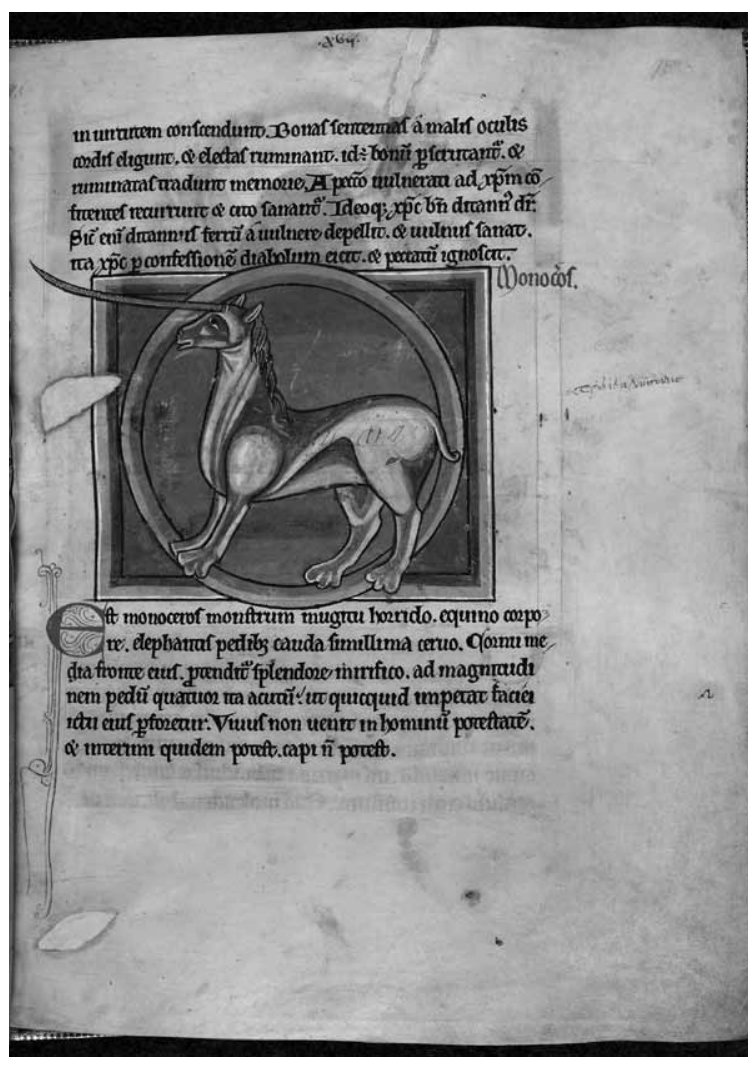

2: Einhorn. Bestiarium, Südengland, erste Hälfte des 13. Jahrhunderts.

nach Süden als es das heute tut, nachdem die Jagd es über Jahrhunderte verdrängt hat. Drei frühere Narwalzahnobjekte sind aus Kirchen bekannt. ${ }^{8}$ Zwei davon bilden offensichtlich ein Paar. Sie wurden möglicherweise um 1120-1140 in England mit Metallapplikationen und Schnitzereien, die von Menschen und Tieren bewohnte Ranken zeigen, verziert, und könnten als Kerzenträger gedient haben. ${ }^{9}$ 入 Abb. 3, 4

6 Guido Schönberger: Narwal-Einhorn. Studien über einen seltenen Werkstoff. In: Städel-Jahrbuch, 9, 1935-1936, S. 168-247, hier 196, situiert die Wende um 1200. Vgl. auch Jürgen Werinhard Einhorn: Spiritalis unicornis. Das Einhorn als Bedeutungsträger in Literatur und Kunst des Mittelalters (1976), München 1998.

7 Jean Malaurie: Die letzten Könige von Thule. Leben mit den Eskimos (frz. Orig. 1955), Frankfurt a. M. 1979.

8 Aleksander Pluskowski: Narwhals or unicorns? Exotic animals as material culture in medieval Europe. In: European Journal of Archaeology, 7, 2004, S. 291-313, hier S. 302f. (dort auch weiterführende Literatur zum Handel).

9 Das eine wird in den National Museums in Liverpool aufbewahrt. Zum anderen im Victoria \& Albert Museum in London. Paul Williamson: Medieval Ivory Carvings. Early Christian to Romanesque, London 2010, S. 382-387. 


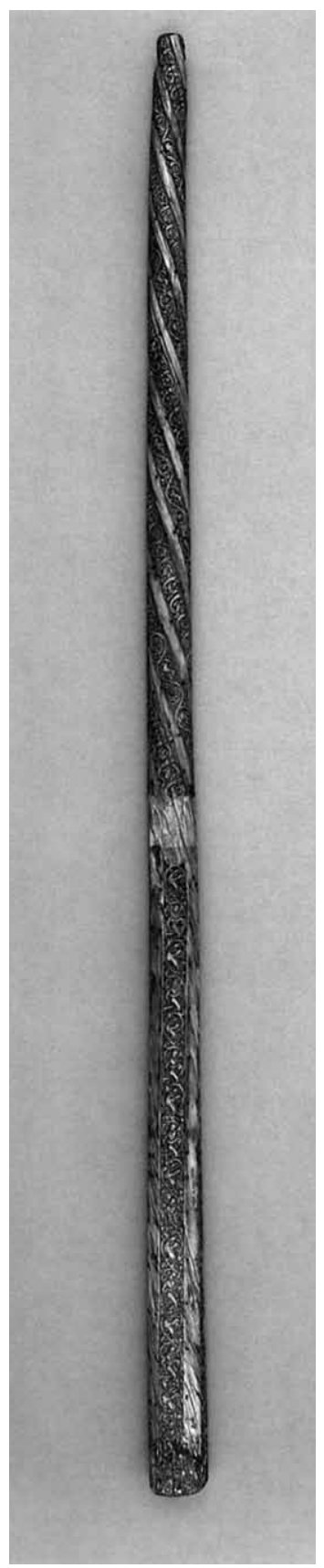

3: Geschnitzter Narwalzahn, England, ca. 1120-1140.
Das dritte bildet den Schaft eines Krummstabes aus dem Salzburger Dom in Österreich und wird auf das Ende des 12. Jahrhunderts datiert. Diese drei Zähne wurden offenbar als Rohmaterial verwendet, wie ein Elfenbein besonders ansprechender Form, ohne eindeutigen Verweis auf ihren tierischen Ursprung. Der Brügger Zahn dagegen, so wie Thomas von Cantimpré ihn erwähnt, hatte wohl als deklariertes Einhorn-Horn eine ganz andere Funktion. Wie war es dazu gekommen? Die Interessen unseres Hauptzeugen liefern dazu manche Informationen.

Thomas war zunächst ein Gelehrter, der in den dominikanischen Schulen von Köln und Paris studiert hatte und gegen 1244, nach etwa fünfzehnjähriger Materialsammlung, ein „Buch über die Natur der Dinge“ vorlegte. Dieses Liber de natura rerum fasste die damaligen Kenntnisse über den Mensch und die Tieren, die Pflanzen und die Steine, die Sterne und das Wetter zusammen. Es war eine belehrende und unterhaltsame naturwissenschaftliche Einleitung zur Wissenschaft des Göttlichen, aus der die Prediger schöpfen sollten. Thomas erklärt dort nach einer Tradition, die auf den im 2. Jahrhundert verfassten Physiologus zurückgeht, dass das Einhorn ein mächtiges und wildes Tier mit einem einzigen Horn sei, das symbolisch für Christus stehe und nur durch eine Jungfrau gezähmt werden könne, wie seine Mutter Maria eine war, bis Jäger es fangen würden, in einem die Inkarnation figurierenden Moment. ${ }^{10}$ Nachdem Thomas 1246 in das Dominikanerkloster zu Löwen zurückgekehrt war, wandte er sich von der Wissenschaft ab und verstärkt der Seelsorge zu. Zwischen 1256 und 1263 kompilierte er das „Buch der Bienen“ (Liber der apibus), ein Kompendium rhetorisch geschickter Geschichten, welche zur Erbauung der als Bienenstock aufgefassten Gesellschaft der Christen gedacht waren. Das im Mittelalter wichtige Thema der Jungfräulichkeit erklärte er am Beispiel der Einhornjagd, wobei er auf sein Liber de natura rerum verwies. Thomas fügte aber in dem neuen Kontext noch hinzu, dass die sich als Köder anbietende junge Frau das in ihrem Schoß Zuflucht suchende wilde Tier an seinem prächtigen Attribut packen würde und dass ein solches, sieben Fuß langes Horn in einer Kirche zu Brügge, also etwa

10 Thomas Cantimpratensis: Liber de natura rerum, 1. 4, c. 104, hg. v. Helmut Boese, Band 1, Text, Berlin/New York 1973, S. 168. 
hundert Kilometer entfernt von dem Löwener Kloster, von jeder und jedem bestaunt werden könne.

Die Vorteile eines materialisierten und ausgestellten Einhorn-Horns liegen für einen Prediger auf der Hand. Vor einem solchen Objekt und mithilfe des narrativen Details des Anfassens des Horns, das bald auch viele Bilder veranschaulichen würden, ${ }^{11}$ konnte er die Imagination seiner Zuhörer auf effizientere Weise anfeuern, um die Freuden der Jungfräulichkeit begreifbar werden zu lassen. Das exemplum wurde beeindruckend plastisch vom Exemplar gestärkt. Möglicherweise hatte bereits etwas früher die Vermittlung ehemals gelehrten, klerikalen Wissens zur Erfindung des Einhorn-Horns geführt. Diese mag nach dem Zeugnis der Einhornbilder in England stattgefunden haben. Es wäre dann wohl kein Zufall, dass Philippe de Thaon, der wahrscheinlich dem dortigen königlichen Hof nahe stand, bereits in der ersten

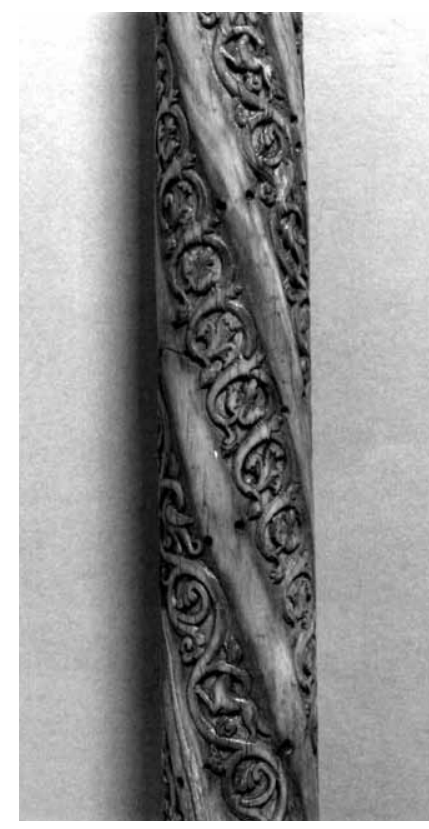

4: Detail aus Abb. 3. Hälfte des 12. Jahrhunderts das erste moralisierte Tierbuch (bzw. Bestiarium) in einer Volkssprache verfasst hatte. ${ }^{12}$ Jedenfalls ist der Ausdruck eines abstrakten Inhalts durch eine konkrete Erfahrung ein typisches didaktisches Verfahren der neuen Predigtkunst des 13. Jahrhunderts, die Thomas von Cantimpré bestens vertrat. ${ }^{13}$ In diesem Fall sollte eine neue Rhetorik des Bewunderns der Natur in die Wunder Gottes einführen. ${ }^{14}$ Die Fokussierung auf das Horn wirkte so gut, dass ab dem 14. Jahrhundert die italienische und die französische Volkssprache das Tier nicht mehr „Ein Horn“ nannten, sondern mit licorno und licorne „Das Horn“ par excellence. ${ }^{15}$

Was war nun echt oder unecht, richtig oder falsch am Einhorn-Horn-Präparat? Echt war das Ding an sich mit seinen Eigenschaften: Das glatte und sanfte, weißlich durchscheinende, bei Kontakt warme Elfenbein, dass schon im biblischen „Hohelied“ Vorstellungen begehrter Haut hervorrief, ${ }^{16}$ seine Härte, seine Spira-

11 Vgl. Einhorn (s. Anm. 6), S. 204.

12 Shannon Hogan Cottin-Bizonne: Une nouvelle édition du „Bestiaire“ de Philippe de Thaon, Chapel Hill 2003.

13 Jacques Le Goff, Jean-Claude Schmitt: Au 13e siècle. Une Parole nouvelle. In: Jean Delumeau (Hg.): Histoire vécue du peuple chrétien, Toulouse 1979, S. 257-278; Nicole Bériou: L'avènement des maîtres de la Parole. La prédication à Paris au XIIIe siècle, Paris 1998.

14 Caroline Walker Bynum: Wonder. In: American Historical Review, 102/1, 1997, S. 1-26.

15 Odell Shepard: The Lore of the Unicorn (1930), New York 1993, S. $141 \mathrm{f}$.

16 „Sein Leib ist wie reines Elfenbein“, „Dein Hals ist wie ein Turm von Elfenbein“: Hld 5,14 und 7,5. 


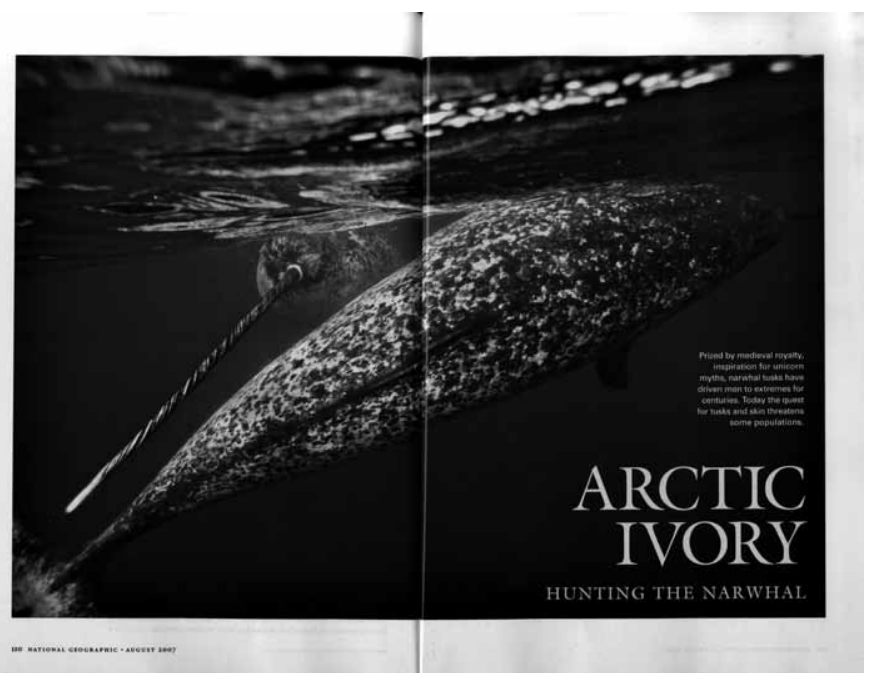

5: Narwale, Paul Nicklen, National Geographic Magazine, 2007. len und die schwindelerregende Größe, als ob es unendlich wachsen und sich in die Luft bohren wollte. Dauerhaft in die Kirche versetzt und mit dem nötigen Kommentar versehen, konnte dieses Ding der Tugendlehre dienen. Bald wurde es auch zum Gegenstand naturwissenschaftlicher Erkenntnis bzw. der neu aufkommenden philosophia naturalis. So berichtet bereits

Albertus Magnus (ca. 1200-1280), in seinem um 1270 fertiggestellten Buch „Über die Tiere“ (De Animalibus), ein über zehn Fuß langes Einhorn-Horn persönlich gemessen zu haben. ${ }^{17}$ Albertus war in Köln der Lehrer von Thomas gewesen, von dem er wiederum Vieles übernahm. ${ }^{18}$ Er hatte aber auch selbst Walfänger der Nordsee befragt. ${ }^{19}$ „Falsch“ dagegen war die Zuschreibung des Dinges an das Einhorn - ein seit Plinius schriftlich bezeugter Vierfüßler aus Indien, ursprünglich wohl ein ungenau beschriebenes Nashorn - statt an den Wal aus dem Norden. Nicht, dass dieser völlig unbekannt gewesen wäre: Thomas von Cantimpré übernimmt aus einem wenig älteren Buch die Beschreibung eines einhörnigen Seemonstrums, das Schiffe hätte durchbohren können, wäre es nur nicht so langsam gewesen. ${ }^{20}$ Die Verbindung zwischen diesem entfernten Ungeheuer und dem schönen Gegenstand war manchem Seemann sicher klar - und zwar so, dass einer nach überwundener Furcht das Objekt als Ex-voto bis nach Brügge oder Lincoln gebracht haben mag. Die gelehrten Klerikern sahen jedoch diesen Bezug nicht mehr, wohl weil die beiden Informationen sie getrennt erreichten, und wohl auch, weil dies damals nur wenig Sinn ergeben hätte.

17 Albertus Magnus: De Animalibus, 1. 12, tract. 3, c. 7, hg. v. Hermann Stadler, 2 Bände, Münster 1916-1920, Band 1, S. 890.

18 John Block Friedman: Albert the Great's Topoi of Direct Observation and his Debt to Thomas of Cantimpré. In: Peter Binkley (Hg.): Pre-Modern Encyclopaedic Texts, Leyden 1997, S. 379-392.

19 Laurence Moulinier: Les baleines d'Albert le Grand. In: Médiévales, 22-23, 1992, S. 117-128.

20 Thomas Cantimpratensis: Liber de natura rerum (s. Anm. 10), 1. 6, c. 35, S. 243. Vgl. S. 4 zum anonymen „Liber rerum“. Albertus übernimmt die Information: De Animalibus (S. Anm. 17), Band 2, S. 1540 . 
Das Einhorn-Horn war kein Horn von Nichts, auch wenn es keine Einhörner gibt, denn es war etwas anderes, nur Vielen etwas Unbekanntes - so zumindest aus unserer Perspektive. Doch was erfahren wir noch, wenn wir heute auf das Mittelalter blicken? Johannes Grave hat im Bezug auf Präparate an die theoretische Erkenntnis erinnert, dass Bil-

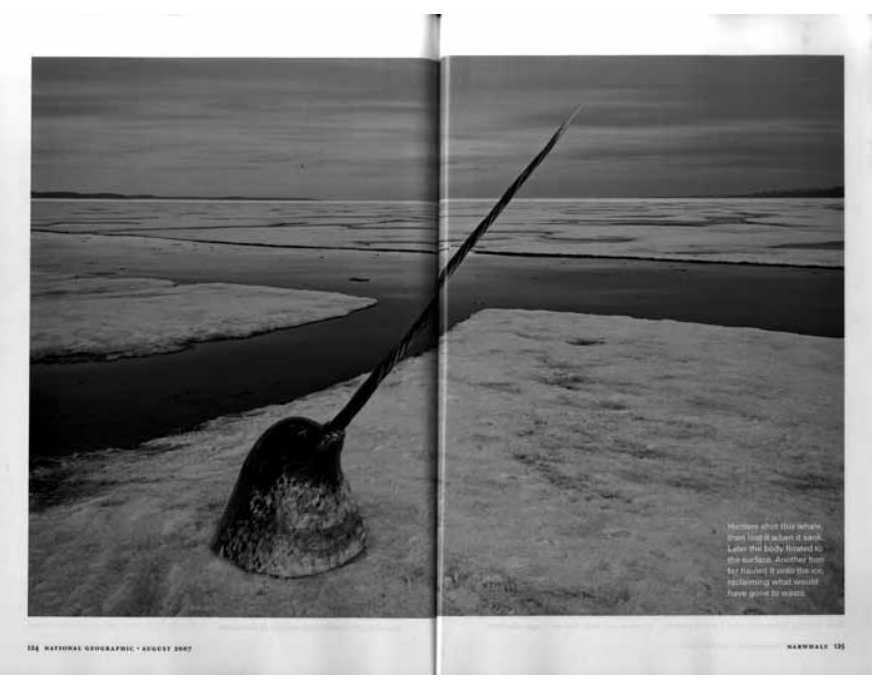

6: Narwaljagd, Paul Nicklen, National Geographic Magazine, 2007. der sich durch eine Differenz zwischen Darstellendem und Dargestelltem auszeichnen. Im Falle der Präparate jedoch, dieser „,Bilder“ ihrer selbst“, schiene zunächst keine Differenz gegeben zu sein - jedenfalls in der naturwissenschaftlichen Auffassung des Präparats, welche die Präparierung als möglichst neutralen Akt darstellt. Bildtheoretisch müsste demnach gefragt werden, „ob die Differenz des Bildes zu dem in der bildlichen Darstellung Gemeinten materiell realisiert sein muss oder auch rein relational gedacht werden kann“. ${ }^{21}$ Der Fall des zum Einhorn-Horn präparierten Narwalzahns macht geradezu evident, dass die Versetzung eines Objektes von einem Kontext in einen anderen freilich ein technischer, aber zugleich und notwendigerweise auch ein kultureller Akt ist, der bei aller Bewahrung der materiellen Identität zwangsläufig eine Differenz produziert. So ist jede Präparation ein Bildgebungsprozess. Als bewusster Transfer eines Dinges von einem Punkt zum anderem, im Raum oder in der Zeit, ist die Präparation eigentlich eine ziemlich banale Operation, die jede Sachkultur mitgestaltet: Die Dinge um uns kommen fast immer von woanders, was ihren jeweiligen Status prägt. Es könnte daher sein, dass der Präparat-Begriff im Bereich der Ding- bzw. Bildwissenschaft eine produktive Karriere vor sich hat. Ein Sonderstatus des wissenschaftlichen Präparats ist aber nicht begründet.

Wissenschafts-, Bild- und Kulturgeschichte mögen in ihrer Beschäftigung mit dem Präparat einen Relativierungskeil in einer ungenügend reflektierten Praxis der

21 Johannes Grave: Nur die Sache selbst? Das Präparat als Grenzfall des Bildes. In: Gottfried Böhm, Sebastian Egenhofer, Christian Spies (Hg.): Zeigen. Die Rhetorik des Sichtbaren, München 2010, S. 141-156, hier 152 . 
Naturwissenschaften geschlagen haben. Ist das aber die Frage? Entscheidender ist jedoch, welche gesellschaftlichen und ökologischen Situationen konfrontiert werden müssen und wie dabei Antworten konzipiert werden können. Kehren wir zu unserer Fallstudie zurück. Die Narwale werden heute mit Jagdwaffen abgeschossen. Die Inuits verkaufen die erhaltenen Zähne im internationalen Handel, um eine moderne Lebensweise zu finanzieren. Der studierte Biologe, Wildlife-Fotograf und Journalist Paul Nicklen, der sich auf die Visualisierung der Konsequenzen der globalen Erwärmung in der Arktis spezialisiert hat, veröffentlichte die bisher eindrucksvollsten Bilder dieser Massenjagd und des Narwals überhaupt. ${ }^{22}$ ^ Abb. 5, 6 Den Naturwissenschaftlern bleibt das Tier jedoch schwer zugänglich. Es ist bis jetzt unklar, welche Funktion dieser seltsame Zahn eigentlich hat. Er könnte als sexuelles Ornament, aber auch als feinfühliges Organ dienen. ${ }^{23}$ Noch ist keine quantitative Einschätzung der Narwalbevölkerung gelungen, die zur Bestimmung wissenschaftlich fundierter Fangquoten dienen könnte. Deren Durchsetzung erweist sich als politisch schwierig. Fest steht jedoch, dass auf der Seite der Kunden das alte Phantasma des Einhorns nach wie vor als Antrieb fungiert. Angesichts der Komplexität der Bedrohung für das Tier und für die Gesellschaft erscheinen also Untersuchungen, Aufklärung und Überzeugung an allen Fronten, sowohl zum Narwal als auch über das menschliche Verhalten, gleichermaßen angebracht. Obwohl das Einhorn-Horn schon im 17. Jahrhundert zum Narwalzahn zurückerklärt worden ist, wurde die Frage nach der Genese dieser Assoziation nie gestellt, wahrscheinlich weil die Faszination des Unbekannten doch noch nachwirkte. Eine Antwort darauf gegeben zu haben, dürfte ein bescheidener Beitrag in einer Angelegenheit sein, die schließlich als exemplum zu veranschaulichen vermag, dass die Präparation von Tieren und sonstigen natürlichen Dingen ein weitreichender und keineswegs neutraler Akt ist.

22 Paul Nicklen, Paul: Arctic Ivory: Hunting the Narwhal. In: National Geographic Magazine, 212/2, August 2007, S. 110-129.

23 William J. Broad, It's Sensitive. Really. In: New York Times, 13. Dezember 2005. 